\title{
Terapia della sindrome nefrosica idiopatica: ruolo delle tecniche aferetiche
}

\author{
Luigi Moriconi
}

Direttore dell’Unità Operativa Complessa di Nefrologia e Dialisi, Empoli

\begin{abstract}
ROLE OF THERAPEUTIC APHERESIS IN THE TREATMENT OF IDIOPATHIC NEPHROTIC SYNDROME
Abstract. Idiopathic NS (INS) is characterized by diffuse foot process effacement on electron microscopy and minimal changes (minimal change nephropathy [MCN]), or focal segmental glomerular sclerosis (FSGS) on optical microscopy. MCN is the most common form of INS in children and is sensitive to corticosteroid therapy in almost cases. FSGS is more common in adult patients and has a less favourable course. Most of drug resistant patients develop terminal renal failure in 6-8 years. Moreover, FSGS reappears in many cases after renal transplantation. Genetic or secondary forms of INS have been identified and their response to therapy is unpredictable, whereas the recurrent forms in transplanted kidney are considered a more omogeneous group. Sera from patients with FSGS may contain some proteinuric or permeability factors (PFs), which have been partially identified. Corticosteroids and immunosuppressive drugs are the mainstay of the standard therapy. In steroid resistant cases, new therapeutic strategies directed against the action of PFs are under evaluation. The removal of PFs by means of Plasmapheresis or selective aphesis has been associated with proteinuria reduction. In this review we will analyze the preliminary results of the new therapies and the main studies on apheretic treatment in steroid resistant patients affected by INS.
\end{abstract}

Key words: Proteinuria, FSGS, PFs, Plasmapheresis

Conflict of interest: None.

Financial support: None.

Ricevuto: 29 Gennaio 2013; Accettato: 4 Marzo 2013

\section{Definizione e patogenesi}

L'acronimo in lingua inglese INS (Idiopathic Nephrotic Syndrome) definisce due malattie: la Glomerulopatia a Lesioni Glomerulari Minime (Minimal Change Nephropathy, MCN) e la Glomerulosclerosi Focale Segmentale (Focal Segmental Glomerular Sclerosis, FSGS). Se queste due patologie siano da considerare come un'unica entità in fasi diverse di evoluzione o comunque due entità, due "fenotipi" per intenderci, correlate nella patogenesi oppure se siano due malattie distinte è un argomento controverso. In una percentuale di casi, la MCN e, maggiormente, la FSGS sono familiari, legate a una mutazione dei geni NPHS1 e NPHS2 che codificano la sintesi di nefrina e di podocina. Soprattutto per la FSGS sono state identificate possibili eziologie virali o genetiche, oltre a forme secondarie in corso di altre malattie, per cui non è semplice classificare queste glomerulopatie. Le forme ricorrenti nel rene trapiantato costituiscono un gruppo più omogeneo. Due fattori, comunque, sono comuni alla MCN e alla FSGS, anche se maggiormente espressi nella seconda: 1) la lesione glomerulare caratterizzante è a carico dei podociti; 2) è frequente il riscontro di sostanze circolanti, definite fattori di permeabilità (PFs). Inoltre, questi PFs possono essere rimossi con plasmaferesi standard (PEX) o con tecniche di aferesi selettiva, soprattutto Immunoadsorbimento con Proteina A (IAA). Studi iniziati negli anni '70 e proseguiti nei decenni successivi $(1,2)$ hanno dimostrato la presenza di PFs, sulla cui identificazione esistono studi recenti (3). In ogni caso, la presenza di INS impone un trattamento intensivo per indurre la remissione, perché la proteinuria elevata condiziona la sopravvivenza della funzione renale (4): nella storia naturale della malattia, oltre il $50 \%$ dei casi con proteinuria in range nefrosico va in insufficienza renale terminale entro 6-8 anni. Da un esame globale delle statistiche si ricava che la FSGS ricorre nel rene trapiantato con una frequenza prossima al $40 \%$ e che nella metà dei casi porta alla perdita della funzione renale. La recidiva in un eventuale secondo trapianto è più frequente $(5,6)$. Comunque, la presenza dei PFs nel siero dei pazienti candidati al trapianto è ritenuta con buona approssimazione predittiva di recidiva e sono stati proposti schemi di trattamento con metodiche aferetiche per prevenire la recidiva di FSGS nel rene trapiantato (5).

Dati sperimentali e osservazioni cliniche hanno consentito di 
identificare alcune piccole proteine di peso molecolare intorno ai $30 \mathrm{kD}(3)$, capaci di svolgere un'attività permeabilizzante dei capillari glomerulari e, quindi, capaci di indurre proteinuria:

VPF (vascular permeability factor): linfochina prodotta da linfociti $\mathrm{T}$; agisce sulla permeabilità capillare sistemica e glomerulare.

Emopexina: proteasi che attiva la protein chinasi B e il rimaneggiamento del citoscheletro dei podociti; riduce il glicocalice endoteliale.

suPAR (soluble urokinase plasminogen activator receptor): l'induzione di un recettore solubile dell'urokinasi nei podociti attiva la $\beta 3$ integrina sui podociti con fusione dei processi pedicolari.

CLC-1 (cardiotrophin like cytokine 1): appartiene alla famiglia dell'IL-6 e riduce l'espressione di Nefrina dai glomeruli e dai podociti in coltura. Viene isolata dalla Cromatografia per affinità con il galattosio.

Uno o più di questi fattori di permeabilità sono stati isolati dal plasma di pazienti con MCN, FSGS e FSGS ricorrente nel trapianto. La letteratura, negli ultimi anni, attribuisce un'importanza prevalente alla presenza del suPAR nel siero dei pazienti con INS, particolarmente quando ricorrente nel trapianto (7).

\section{Strategie terapeutiche standard e nuove}

Sulla base delle recenti acquisizioni sulla patogenesi della INS, le strategie terapeutiche sono mirate a interferire a vari livelli con l'azione proteinurica dei PFs (Tab. I).

Sappiamo che la terapia con dosi elevate di corticosteroidi somministrati per alcuni mesi può ottenere un elevato grado di remissione parziale o totale e che, aggiungendo un inibitore della Calcineurina nei casi steroido-dipendenti o steroido-resistenti, la risposta arriva fino al $70 \%$ dei casi trattati. Nei casi resistenti, le nuove terapie farmacologiche non hanno, fino a oggi, studi che dimostrino un'analoga efficacia; sul Rituximab esistono alcuni studi, prevalentemente non randomizzati prospettici, su casistiche pediatriche, che forniscono risultati promettenti, ma non conclusivi (8). Vi sono alcuni casi aneddotici di risultato favorevole nella FSGS ricorrente nel trapianto, associando Rituximab e trattamento aferetico. Sono pubblicati alcuni report preliminari e sono ancora in corso alcuni trial del FONT (novel therapies for resistant FSGS) che valutano l'efficacia dell'associazione di anti-TNF (adalimumab) con rosiglitazone o galattosio $(9,10)$.

\section{Revisione dei principali studi su plasmaferesi e immunoadsorbimento}

Dal 1992 fino ai primi anni 2000, sono stati pubblicati alcuni importanti studi che hanno evidenziato l'efficacia dell'aferesi terapeutica sia come PEX che come IAA. Mary Artero (11), nel 1992, descriveva l'induzione di remissione in 25 casi di FSGS recidivante nel rene trapiantato, aggiungendo PEX alla terapia immunosoppressiva, e ipotizzava il ruolo di fattori proteinurici circolanti. Due anni dopo, Jacques Dantal e altri del gruppo di Nantes (12) pubblicavano un confronto di efficacia nella riduzione della proteinuria in pazienti trapiantati, fra il trattamento con PEX e IAA; i risultati mostravano una remissione con le due metodiche, migliore con IAA. Nel 1996, Virginia Savin et al., in un importante studio pubblicato sul NEJM (13), dimostravano che i PFs circolanti si associavano frequentemente alla recidiva di FSGS. La loro rimozione mediante PEX si associava alla remissione della proteinuria. Nel 1998, Haas (14) pubblicava su NDT una casistica di 8 pazienti, 5 con FSGS nei reni nativi e 3 con recidiva nel trapianto, tutti trattati con IAA: 4 ottenevano la riduzione di oltre il $50 \%$ della proteinuria, tutti con positività del test di permeabilità glomerulare. Nel 1999, un importante studio multicentrico che ho già ricordato (5) evidenziava come, in una casistica di 25 bambini con recidiva di FSGS nel trapianto, la presenza di fattori circolanti di permeabilità aveva un valore predittivo di recidiva e che il trattamento con PEX associato alla terapia con ciclofosfamide induceva la remissione nella maggioranza dei casi. Nel 2001, il nostro gruppo di Pisa pubblicava i risultati del trattamento di 7 pazienti con FSGS dei reni nativi e del rene trapiantato (15): negli anni successivi si aggiungevano 3 casi per un totale di 10: 7 con malattia dei reni nativi e 3 con recidiva nel rene trapiantato, 7 trattati con IAA e 3 con PEX; 2 su 3 casi con recidiva e 3 su 7 con FSGS dei reni nativi ottenevano remissione. Il ciclo di terapia aferetica era costante nei pazienti

TABELLA I - OBIETTIVI DEL POSSIBILE INTERVENTO TERAPEUTICO NELLA INS

\begin{tabular}{ll}
\hline Tipo di strategia terapeutica & Terapia già realizzabile o possibile \\
\hline $\begin{array}{l}\text { Ridurre i livelli plasmatici dei PFs di qualunque origine } \\
\text { (anche prodotti da linfociti T e citochine) }\end{array}$ & $\begin{array}{l}\text { Farmaci immunosoppressori, anticorpi monoclonali, assunzione di sostanze } \\
\text { "leganti” o bloccanti l'azione (galattosio), PEX, aferesi selettiva, IAA }\end{array}$ \\
Proteggere l'endotelio da sostanze che aumentano la permeabilità (VPF) & TGF- beta-1 e VEGF avrebbero questa azione
\end{tabular}

Mantenere la struttura fisiologica del podocita, bloccare l'alterazione del glicocalice

Terapia aspecifica, tendente a modificare la permeabilità capillare attraverso variazioni emodinamiche e inibitrice dell'evoluzione verso la fibrosi
Dosi elevate di corticosteroidi, inibitori della calcineurina, antiossidanti. Anche il Rituximab e l'aferesi avrebbero questa azione

ACE inibitori, sartanici, anticorpi anti TNF, rosiglitazone, farmaci ipolipemizzanti come le statine 
trattati, sulla base di un protocollo terapeutico da noi adottato regolarmente (16), che prevede 10 sedute in 4 settimane e poi un percorso di graduale riduzione se i risultati del primo periodo intensivo sono soddisfacenti. Il trattamento era, comunque efficace sui livelli di PFs, ma la remissione clinica, in termini di riduzione della proteinuria e miglioramento della funzione renale, poteve correlarsi oppure no alla riduzione dei fattori di permeabilità. Gli Autori concludevano che la prognosi del risultato dell'aferesi dipende da alcune variabili, fra cui particolarmente rilevanti:

1 - presenza di PFs circolanti;

2 - prevalenza di lesioni suscettibili di regressione nello score istologico renale;

3 - adeguatezza dell'aferesi (volume di plasma, numero di sedute);

4 - mantenimento della terapia immunosoppressiva durante il ciclo aferetico.

Il meccanismo d'azione del trattamento aferetico nella INS non è completamente chiarito e alcuni Autori, in uno studio pubblicato su JASN nel 1999 (17), hanno ipotizzato un effetto antiproteinurico dell'IAA dovuto alla rimozione di soluti che alterano la permeabilità o l'emodinamica del glomerulo, definendolo "aspecifico", in quanto rilevato in nefropatie di eziologia e patogenesi varie.

La concezione che una tecnica di rimozione abbia un effetto aspecifico di riduzione della proteinuria è interessante, perché noi conosciamo già un effetto aspecifico antiproteinurico di una classe di farmaci, gli inibitori della Calcineurina, ai quali viene riconosciuta una grande efficacia antiproteinurica legata soprattutto all'azione enzimatica di inibizione della defosforilazione della sinaptopodina, che ha come effetto la stabilizzazione del citoscheletro del podocita (18).

La review pubblicata nel Gennaio del 2010 da Claudio Ponticelli (6) focalizza i fattori di rischio per la recidiva nel rene trapiantato, la patogenesi con riguardo anche alla presenza di PFs, i rapporti con la mutazione del gene NPHS2, il trattamento farmacologico e la possibile efficacia dei trattamenti aferetici. Da una metanalisi di 12 studi condotti su una casistica pediatrica ( 70 bambini) e di 10 condotti su pazienti adulti (77 casi), riguardanti l'associazione di PEX o IAA ai farmaci tradizionali, emerge che 49 bambini (70\%) e 49 adulti (63\%) ottenevano la remissione della FSGS recidiva nel trapianto.

\section{LDL aferesi nel trattamento della sindrome nefrosica idiopatica}

In letteratura ci sono molti studi riferiti al trattamento della INS, con la LDL aferesi (LDL-A), e la metodica maggiormente impiegata è la plasmaseparazione seguita dall'adsorbimento su colonne di Destrano Solfato. Gli Autori più impegnati in questa attività sono giapponesi. Nel primo studio importante (19), gli Autori esaminano gli effetti dell'aggiunta della LDL-A in 17 casi con ipercolesterolemia e FSGS alla terapia steroidea, confrontati con 10 casi simili, trattati con la sola terapia steroidea. Il gruppo con terapia combinata ottiene una remissione della proteinuria in un tempo significativamente inferiore rispetto al gruppo in monotera- pia steroidea. Questo effetto antiproteinurico è associato a un'altrettanto rapida normalizzazione della lipemia nel gruppo con terapia combinata.

Gli stessi Autori (20), alcuni anni dopo, hanno pubblicato uno studio multicentrico retrospettivo sull'outcome a 5 anni di 41 pazienti trattati con LDL-A e prednisone, una parte dei quali era stata descritta nel precedente studio. A 2 anni e a 5 anni il $62 \%$ e $1 ' 87 \%$ dei casi era in remissione completa o parziale. Fattori determinanti del risultato positivo sono la precocità del trattamento aferetico dopo la comparsa della sindrome nefrosica e la drastica riduzione dei livelli del colesterolo.

Nel 2003, sull'AJKD (21), viene esaminato l'outcome clinico di 11 bambini con FSGS farmaco-resistente, trattati con un primo ciclo di LDL-A in monoterapia e, successivamente, con un secondo ciclo di LDL-A in associazione con prednisone. Mentre la sola LDL-A non ottiene la remissione della proteinuria, pur ottenendo la riduzione della lipemia, durante il secondo ciclo viene ottenuta la remissione della proteinuria nella maggioranza dei casi.

Non è possibile trarre conclusioni sicure da questi studi sull'efficacia della LDL-A, anche perché si tratta di casistiche di FSGS nei reni nativi, che sono forme meno omogenee di quelle recidive nel trapianto e con patogenesi che può essere multifattoriale. Tuttavia è interessante rilevare che, comunque, l'effetto antiproteinurico non sembra direttamente collegato alla normalizzazione dei lipidi plasmatici che si verifica in ogni caso. Inoltre, la LDL-A sembra non essere efficace in monoterapia, ma solo associata allo steroide, in forme già definite steroido-resistenti, come se la rimozione dei soluti (lipoproteine, ma anche altri soluti) rendesse i pazienti sensibili alla terapia farmacologica.

\section{Conclusioni}

È ormai generalmente riconosciuto che il meccanismo comune della proteinuria e della progressione del danno renale è l'alterazione della struttura del podocita. Sono presenti in letteratura molti studi fortemente indicativi di efficacia antiproteinurica anche in forme farmaco-resistenti di INS, nei reni nativi e, soprattutto, nei reni trapiantati, dell'uso di tecniche aferetiche massive o selettive. Soprattutto nel trapianto renale in soggetti affetti precedentemente da FSGS, il trattamento preventivo con aferesi può contribuire a ridurre l'incidenza della recidiva. Inoltre, l'instaurazione precoce dell'aferesi in caso di insorgenza di proteinuria indice di recidiva contribuisce a indurre la remissione. La recente identificazione di alcuni PFs che danneggiano il podocita e la conseguente possibilità di migliorare il razionale teorico della rimozione totale o selettiva di plasma aprono la prospettiva di impiegare le tecniche di terapia aferetica in modo razionale, correlando i risultati con le variazioni dei fattori biologici. Dovrebbe essere invertita la tendenza attuale che è di utilizzo molto scarso di queste tecniche nella INS: $5 \%$ delle indicazioni nelle malattie renali negli ultimi 3 anni (22). Una valutazione più attendibile di questi trattamenti richiede che i centri di nefrologia ne implementino l'utilizzo, costruendo studi multicentrici prospettici anche in un contesto multidi- 
sciplinare (centri trasfusionali, pediatria, centri trapianto) e cercando di omogeneizzare per quanto possibile le casistiche e i trattamenti. Si apre anche la prospettiva di associare PEX e IAA con le nuove strategie terapeutiche, oltre che con la terapia farmacologica standard.

\section{Riassunto}

La Sindrome Nefrosica Idiopatica (Idiopathic Nephrotic Syndrome, INS) ricorre essenzialmente in presenza di due glomerulopatie: la MCN (Minimal Change Nephropathy) e la FSGS (Focal Segmental Glomerular Sclerosis). La prima ha un decorso più benigno ed è più frequente nei bambini, mentre la seconda ha un decorso più severo, può portare a Insufficienza Renale Cronica Terminale e può recidivare nel trapianto. Soprattutto per la FSGS sono state identificate possibili eziologie virali o genetiche, oltre a forme secondarie in corso di altre malattie, per cui non è semplice classificare queste glomerulopatie. Le forme ricorrenti nel rene trapiantato costituiscono un gruppo più omogeneo. I fattori che sembrano essere comuni alla MCN e alla FSGS, anche se maggiormente espressi e studiati nella seconda, sono la lesione glomerulare caratterizzante a carico dei podociti, e il frequente riscontro di sostanze circolanti, definite fattori di permeabilità (PFs), capaci di indurre proteinuria. Corticosteroidi e Immunosoppressori sono la terapia standard della INS. Tuttavia, la presenza di casi farmaco-resistenti e l'identificazione di alcuni PFs circolanti hanno consentito di utilizzare nuove terapie dirette a bloccare la sintesi o l'azione di queste molecole e hanno fornito un ulteriore razionale alla loro rimozione mediante plasmaferesi convenzionale (PEX) o aferesi selettiva.

Parole chiave: Proteinuria, FSGS, PFs, Plasmaferesi

Dichiarazione di conflitto di interessi: L'Autore dichiara di non avere conflitto di interessi.

Contributi economici degli autori: L'Autore dichiara di non aver ricevuto sponsorizzazioni economiche per la preparazione dell'articolo.

Indirizzo degli Autori:

Dr. Luigi Moriconi

Direttore dell'Unità Operativa Complessa

di Nefrologia e Dialisi

Ospedale degli Infermi, San Miniato

Azienda USL 11, Empoli

nefrosm@tin.it

\section{Bibliografia}

1. Hoyer JR, Raij L, Vernier RL, Simmons RL, Najarian JS, Michael AF. Recurrence of idiopathic nephrotic syndrome after renal transplantation. Lancet 1972; 2: 343-8.

2. Koyama A, Fujisaki M, Kobayashi M, Igarashi M, Narita M. A glomerular permeability factor produced by human $\mathrm{T}$ cells hybridomas. Kidney Int 1991; 40: 453-60.

3. McCarthy ET, Sharma M, Savin VJ. Circulating permeability factors in idiopathic nephrotic syndrome and focal segmental glomerulosclerosis. Clin J Am Soc Nephrol 2010; 5: 2115-21.

4. Korbet SM, Schwartz MM, Lewis EJ. Primary focal segmental glomerulosclerosis: clinical course and response to therapy. Am J Kidney Dis 1994; 23 (6): 773-83.

5. Dall'Amico R, Ghiggeri GM, Carraro M, et al. Prediction and treatment of recurrent focal segmental glomerulosclerosis after renal transplantation in children. Am J Kidney Dis 1999; 34: 1048-55.

6. Ponticelli C. Recurrence of focal segmental glomerular sclerosis (FSGS) after renal transplantation. Nephrol Dial Transplant 2010; 25: 25-31.

7. Wei C, El Hindi S, Li J, et al. Circulating urokinase receptor as a cause of focal segmental glomerulosclerosis. Nat Med 2011; 17 (8): 952-60.

8. Pradhan M, Furth S. Rituximab in steroid-resistant nephrotic syndrome in children: a (false) glimmer of hope? J Am Soc Nephrol 2012; 23: 975-8.
9. Peyser A, Machardy N, Tarapore F, et al. Follow-up of phase I trial of adalimumab and rosiglitazone in FSGS: III. Report of the FONT study group. BMC Nephrol 2010; 11: 2.

10. Trachtman H, Vento S, Gipson D, et al. Novel therapies for resistant focal segmental glomerulosclerosis (FONT) phase II clinical trial: study design. BMC Nephrol 2011; 12: 8 .

11. Artero M, Biava C, Amend W, Tomlanovich S, Vincenti F. Recurrent focal glomerulosclerosis: natural history and response to therapy. Am J Med 1992; 92: 375-83.

12. Dantal J, Bigot E, Bogers W, et al. Effect of plasma protein adsorption on protein excretion in kidney transplant recipients with recurrent nephrotic syndrome. N Engl J Med 1994; 330: 7-14.

13. Savin VJ, Sharma R, Sharma M, et al. Circulating factor associated with increased glomerular permeability to albumin in recurrent focal segmental glomerulosclerosis. N Engl J Med 1996; 334: 878-83.

14. Haas M, Godfrin Y, Oberbauer R, et al. Plasma immunoadsorption treatment in patients with primary focal and segmental glomerulosclerosis. Nephrol Dial Transplant 1998; 13: 2013-6.

15. Moriconi L, Lenti C, Puccini R, et al. Proteinuria in Focal Segmental Glomerulosclerosis: role of circulating factors and therapeutic approach. Ren Fail 2001; 23 (3-4): 533-41.

16. Moriconi L, Passalacqua S, Pretagostini R, et al. Apheresis in primary focal segmental glomerulosclerosis of native and transplanted kidneys: a therapeutic protocol. J Nephrol 2000; 13 (5): $347-51$. 
17. Esnault VL, Besnier D, Testa A, et al. Effect of protein A immunoadsorption in nephrotic syndrome of various etiologies. J Am Soc Nephrol 1999; 10: 2014-7.

18. Mathieson PW. Proteinuria and immunity--an overstated relationship? N Engl J Med 2008; 359 (23): 2492-4.

19. Muso E, Mune M, Fujii Y, et al. Significantly rapid relief from steroid-resistant nephrotic syndrome by LDL apheresis compared with steorid monotherapy. Nephron 2001; 98 (4): 408-15.

20. Muso E, Mune M, Yorioka N, et al. Beneficial effects of low- density lipoporotein apheresis (LDL-A) on refractory nephrotic syndrome (NS) due to focal glomerulosclerosis (FGS). Clin Nephrol 2007; 67 (6): 341-4.

21. Hattori M, Chikamoto H, Akioka Y, et al. A combined low density lipoporotein apheresis and prednisone therapy for steroid-resistant primary focal segmental glomerulosclerosis in children. Am J Kid Dis 2003; 42 (6): 1121-30.

22. Clark WF. Plasma Exchange for renal disease: evidence and use 2011. J Clin Apher 2012; 27 (3): 112-6. 